Revista de Psicología Vol. 35 (1), 2017 (ISSN 0254-9247)

\title{
Representaciones sociales de líderes institucionales acerca del juego en casinos mexicanos
}

\author{
Rebelín Echeverría Echeverría ${ }^{1}$, María Teresita Castillo ${ }^{2}$, Nancy Evia ${ }^{3}$, \\ Carlos Carrillo ${ }^{4}$ \\ Universidad Autónoma de Yucatán
}

\begin{abstract}
El objetivo del estudio fue analizar las representaciones sociales de diferentes líderes institucionales en torno a los clientes de los casinos, sus motivaciones principales y el papel del casino en la cotidianidad. La metodología fue cualitativa-fenomenológica con entrevistas semiestructuradas. Participaron ocho líderes de instituciones religiosas, políticas, de salud mental y funcionarios gubernamentales. Los resultados evidencian que las mujeres en edad adulta se representan como el colectivo con mayor presencia en los casinos. Todos los participantes representan a las personas jugadoras asociadas al juego patológico y la soledad, con variaciones según la institución que representan. Se discute la pertinencia de generar procesos investigativos que amplíen la concepción de los juegos en casinos e impacten en las políticas públicas en salud y desarrollo socioeconómico.

Palabras clave: representaciones sociales, líderes, juego, casinos.
\end{abstract}

1 Doctora en Psicología por el Centro de Estudios, Clínica e Investigación Psicológica de Mérida, México y profesora titular de la Facultad de Psicología de la Universidad Autónoma de Yucatán. Dirección postal: Calle 18a, num 420, por 31 d y 35, Nueva Alemán, CP 97149, Mérida Yucatán, México. Contacto: rebelin.echeverria@gmail.com y rechever@correo.uady.mx

2 Doctora en Estudios Mesoamericanos por la Universidad de Hamburgo y profesora titular C de la Facultad de Psicología de la Universidad Autónoma de Yucatán. Dirección postal: Calle 25, num. 310, por 8 y 10, San Esteban, Mérida Yucatán, 90149. Contacto: tete.castillo@ correo.uady.mx y castillo.tete@gmail.com

3 Magíster en Psicología Clínica Infantil por la Universidad Autónoma de Yucatán y técnico académico asociado A en la Universidad Autónoma de Yucatán. Dirección postal: Calle 59L, num. 574G, por 112 y 114, col. Bojórquez, Mérida, Yucatán, México. Contacto: nancy. evia@gmail.com

4 Doctor en Psicología por el Centro de Estudios, Clínica e Investigación Psicológica de Mérida, México y profesor de carrera asociado de la Facultad de Psicología de la Universidad Autónoma de Yucatán. Dirección postal: Calle 10b, num. 342, por 37a y 39, Fraccionamiento Real del Sol, Mérida Yucatán, 97144. Contacto: carrillo.trujillo@gmail.com 


\section{Social representations of institutional leaders about gambling in México}

This study aims to analyze the social representations of different institutional leaders regarding the casino customers, their main motivations and role of the casino in daily life. The methodology was qualitative - phenomenological using semi-structured interviews. Participants were eight leaders of religious, political, and mental health institutions, and government officials. The results show that adult women are represented as the group with greater presence in the casinos. Loneliness and pathological gambling are part of the social representation of the players, with variations according to the institution that the leaders represent. The relevance of generating investigative processes that expand the concept of the games in casinos and impact on public health policies and socioeconomic development is discussed.

Keywords: Social representations, leaders, game, casinos.

\section{Representaçóes sociais do líderes institucionais sobre o jogo em cassinos mexicanos}

O objetivo é analisar as representaçôes sociais de diferentes líderes institucionais em torno dos clientes do casino, as suas principais motivaçôes e papel do casino na vida diária. A metodologia foi qualitativa fenomenológica com entrevistas semi-estruturadas. Os participantes foram oito líderes de instituiçóes de saúde, religiosas, políticas, de saúde mentais e funcionários do governo. Os resultados mostram que as mulheres adultas são representados como o grupo com maior presença nos casinos. Todos os participantes representam jogadores pessoas associadas com o jogo patológico e solidão, com variaçóes de acordo com a instituição que representam. A relevância de gerar processos de pesquisa que expandem o conceito de jogo em casinos e impacto na política de saúde pública e do desenvolvimento socioeconômico é discutido.

Palavras-chave: representaçóes sociais, líderes, cassinos. 
Moscovici (1981) señala que las representaciones sociales son la versión contemporánea del sentido común. Las representaciones brindan estabilización del marco de vida de los individuos y los grupos, y que se constituyen como instrumento de orientación en la percepción de situaciones y la elaboración de respuestas. De acuerdo con Jodelet (1991) toda representación social es una forma de conocimiento social, caracterizada por ser socialmente elaborada y compartida; apunta a un fin práctico de organización de la información y de dominio del ambiente sea este material, social o ideal; da orientación a los comportamientos y las comunicaciones y, finalmente, se dirige a establecer una visión de la realidad común a un conjunto social o cultural dado. Esta misma autora argumenta que las representaciones sociales:

...conciernen al conocimiento de sentido común que se pone a disposición en la experiencia cotidiana; son programas de percepción, construcciones con status de teoría ingenua, que sirven de guía para la acción e instrumento de lectura de la realidad; sistemas de significaciones que permiten interpretar el curso de los acontecimientos y las relaciones sociales; que expresan la relación que los individuos y los grupos mantienen con el mundo y los otros; que son forjadas en la interacción y el contacto con los discursos que circulan en el espacio público; que están inscritas en el lenguaje y en las prácticas; y que funcionan como un lenguaje en razón de su función simbólica y de los marcos que proporcionan para codificar y categorizar lo compone el universo de la vida. (Jodelet, 2000, citado por Perera, 2005, p. 47).

Las representaciones sociales reconocen el hecho de que el conocimiento es social en su origen y no el producto de la cognición individual (Wagner \& Flores-Palacios, 2010). La relación epistémica de la persona con un objeto se define y es mediada por las otras personas relevantes. El grupo, a través de su sistema de representaciones, elaborado en el discurso y en los actos de comunicación, es la base a partir de la cual el individuo comprende e interactúa con el mundo. 
De acuerdo con Elejabarrieta (1995) existen tres grandes tipos de objetos capaces de originar un proceso representacional: el primero son las ideas y teorías científicas de corte utilitario en la vida cotidiana. El segundo es la imaginación cultural, los elementos míticos o mágicos relevantes para los grupos sociales en un contexto específico. El tercero, se refiere a las condiciones sociales y acontecimientos significativos de la vida que son polémicos y objeto de la discutibilidad social. En este sentido, consideramos el juego en casinos como objeto capaz de originar un proceso representacional, en el cual los elementos míticos y mágicos cobran relevancia a través del azar y, adicionalmente, resulta ser una práctica de discutibilidad social polémica para diferentes grupos y contextos sociales.

En el contexto contemporáneo, el ocio es requisito para la construcción de una sociedad de consumo. Sin embargo, para el capitalismo, paradójicamente, la propia palabra resulta peligrosa. Al significado de ocio se ha incorporado diferentes representaciones a lo largo de la historia, generalmente se le asocia a lo negativo, lo que se expresa en diversos refranes y máximas como el de "la ociosidad es madre de todos los vicios". Entender la palabra en su sentido original - tal como lo hacían las antiguas sociedades griega o romana-, como tiempo de inactividad, para "no hacer nada", desde la lógica del capitalismo, era incorrecto, pues se considera como un desperdicio de oportunidades de acrecimiento del capital (Fuentes Gómez, 2013).

El ocio entendido como tiempo libre es relativamente reciente y su aparición está unida a los países altamente industrializados; donde el reloj y los horarios imponen una estructura y organización estandarizada para desarrollar todas las actividades de la vida. Se relaciona con la pérdida del tiempo natural, que es sustituido por el tiempo del reloj y la realización de trabajos con los que difícilmente llega a identificarse la persona, pero que son parte de la rutina diaria y que requiere de incluir espacio para estar libre (Fuentes Gómez, 2013). En general podemos decir que el tiempo libre son aquellos períodos de la vida humana en los cuales las personas se sienten libres. Libertad relacionada con un doble significado: libres de y libres para. El ocio es entendido entonces como aquel tiempo en el que actuamos por nuestra propia voluntad, 
Representaciones sociales de líderes institucionales acerca del juego... / Echeverría et al.

por nuestros impulsos y según nuestros deseos e inclinaciones (Fuentes Gómez, 2013). De ahí el tiempo libre pasa a ser en muchas ocasiones, tiempo privado, opuesto al tiempo asignado a ocupaciones y responsabilidades de diversa índole. En la actualidad esta idea de libertad se ha visto modificada por la industria del entretenimiento y diversión, transformándola cada vez más en actividades de esparcimiento que resultan estructuradas y planeadas.

El tiempo libre y el esparcimiento como una construcción social, implica verlos como una creación humana que cambia con el tiempo y está abierta a la interpretación; como hecho histórico, donde cada época marca pautas de conducta sobre las buenas maneras de usar el tiempo libre y de recreación; donde los factores sociales y económicos, así como el ocio como industria, contribuyen grandemente en las posibilidades de uso del mismo. Tradicionalmente el trabajo y el ocio se han planteado como actividades en franca contraposición. Situación que se evidencia en la sociedad contemporánea donde existe una sobrevaloración del trabajo y la productividad, aunada a la devaluación de otras áreas de la vida como el aspecto personal, emocional, espiritual, social y de ocio o esparcimiento. Particularmente, el fenómeno de los juegos de azar, como actividad de ocio, es tan antiguo como la civilización misma y por ello, no puede ser visto como un fenómeno monolítico y aislado del resto de los fenómenos y contextos históricos, sociales, económicos, políticos, religiosos y culturales. El juego en casinos forma parte del entramado social en constante proceso de construcción (Echeverría Echeverría \& Evia Alamilla, 2013).

El tiempo libre y de esparcimiento nos trae beneficios a todos los individuos, grupos sociales y sociedad en general. Aunque la sociedad industrial actual nos ha impuesto una agenda de trabajo cada vez más apretada y que nos amenaza con la escasez del mismo, obliga muchas veces a las personas por un lado a ocuparse en cosas que no les gusta y por otro a emplear de alguna manera parte del tiempo para el descanso y esparcimiento (Fuentes Gómez, 2013). Hay que reconocer que el ser humano requiere del desarrollo de todos los aspectos de su vida, dejando de lado visiones que priorizan solo unas esferas en detrimento de otras (Echeverría Echeverría \& Evia Alamilla, 2013). 
En este contexto, el estudio de las representaciones sociales de los juegos de azar en casino permite reconocer los modos de construcción del pensamiento social y nos aproxima a la visión del mundo de las personas, desde su posicionamiento social, tiempo y espacio. De acuerdo con McGurrin (1992) históricamente el juego ha representado dos perspectivas una con elementos de peligro y otra como pecado con connotaciones de inmoralidad; el juego ha sido simultáneamente común y prohibido. Además de que el mismo autor sostiene que la presencia y manifestaciones de opciones para el juego son dictadas por los caprichos del gobierno y la sociedad.

El juego y el azar han formado y siguen siendo parte fundamental de la necesidad de ciertas personas de retar el destino, de jugar con la suerte y de salir momentáneamente de la vida rutinaria. Se alimentan de los discursos, mensajes y retoricas de las representaciones e imaginarios sociales propios de la sociedad posmoderna, donde la certidumbre y seguridad es cada vez más precaria, y el consumo, ocio, diversión y la riqueza rápida son considerados objetivos legítimos que conducen al éxito, reconocimiento y la felicidad (Fuentes Gómez, 2013).

En la ciudad de Mérida, apenas podemos hablar de una historia de menos de 15 ańos de convivir con los casinos, sin embargo los cambios relacionados con su establecimiento son varios. Como señalan Echeverría Echeverría y Roa Muńoz (2013) y Carrillo Trujillo (2013) los juegos de azar han sido relacionados con el lavado de dinero, las drogas, la politica y la criminalidad y con conductas vinculadas a la adicción de drogas y alcohol. Por otra parte, en México existen pocos datos sobre índices, estadísticas y datos que relacionen la asistencia a los casinos a aspectos como la ludopatía, el ocio, la diversión y la vocación de espacio de interacción lúdico de los casinos. Como consecuencia existen muchos actores sociales que opinan sobre la asistencia a los casinos y del uso y abuso relacionado con ellos. Lo que es un hecho es que como ciudadanos es importante que seamos críticos y reflexivos en cuanto a nuestro papel social y personal ante la llegada de dichos negocios a la escena urbana de nuestra capital (Echeverría Echeverría \& Evia Alamilla, 2013) sobre una temática que es multidimensional. 
El objetivo de este trabajo fue analizar las representaciones sociales que tienen diferentes líderes institucionales en torno a los clientes de los casinos, sus motivaciones principales y el papel del casino en la cotidianidad social.

\section{Método}

\section{Participantes}

Participaron 8 líderes de diferentes instituciones de Yucatán: Una diputada, un sacerdote católico, un pastor cristiano evangélico, un director de asociación civil especializada en atención a las adicciones, un funcionario del gobierno responsable de un programa que da atención a las adicciones, un presidente del Colegio de Psicólogos del Estado y dos profesionales de la psicología con experiencia en la atención a personas con problemas de juego. El criterio en el que se basó la decisión de incluir a dichos actores se sustenta en la idea de que el fenómeno de los casinos debe ser analizado y comprendido desde la representación sociales de actores de los diferentes campos de la vida social, de la política, la religión, la ciencia y las instancias gubernamentales en tanto dichas instituciones funcionan como, en palabras de Foucault (1996), instrumento de normalización que definen un orden moral y político.

\section{Instrumentos}

La técnica principal fue la entrevista semiestructurada. Algunos de los temas abordados en la entrevista fueron las percepciones sobre las razones que motivan a la gente a ir o no ir al casino (estatus, amistades, económico), los eventos significativos vividos en el casino, las acciones de la institución a la que representan con respecto a los casinos, la existencia de programas específicos de atención al juego. Además de las representaciones en torno a las características que atribuyen quienes juegan, su comprensión de dicha práctica, las nuevas prácticas y los cambios en la vida cotidiana al integrarse los casinos a la oferta de ocio de la ciudad, entre otros. Las entrevistas fueron grabadas y transcritas para su posterior análisis. Este último se basó en la estrategia propuesta 
por Rodríguez Gómez, Gil Flores y García Jiménez (1999) que contempla tres grandes operaciones: la reducción de datos, la disposición y transformación de datos y, finalmente, la obtención y verificación de conclusiones.

\section{Procedimiento}

Se realizó la invitación a cada uno de los líderes institucionales explicándoles el objetivo de la investigación y de las entrevistas, la duración de la misma, así como el derecho a declinar su participación y poder retirarse en el momento que lo considere pertinente. También se les aseguró anonimato y se les señalaron los límites de la confidencialidad. Se les brindó espacio para preguntas y, finalmente, se les preguntó si consentían su partición en la investigación de forma oral, al considerar que la investigación no pone a los participantes en un riesgo de responsabilidad de ningún tipo.

Después de obtener una respuesta afirmativa, se acordó con cada uno de los participantes una cita en un espacio con ambiente confortable y se les solicitó su consentimiento para grabar sus voces con el fin de asegurar la recopilación fiel de los datos y así poder proceder al análisis antes mencionado. Todos los participantes accedieron a ser grabados.

\section{Resultados y discusión}

\section{Los clientes: edad, estrato social y religión}

Pensar en los clientes del casino, desde la visión de los diferentes representantes, evidencia cómo el casino hasta ahora en Mérida se representa como un espacio que da cabida a distintos grupos poblacionales sean estos jóvenes, adultos o personas de la tercera edad. Sin embargo, llama la atención, que en la representación social de la mayoría de los participantes las mujeres adultas ocupan un lugar central; sean estas, amas de casa o trabajadoras fuera del hogar, solteras o casadas. En este sentido comenta un funcionario público: “...yo he visto muchísimas, en lo particular mujeres, muchísimas personas, amas de casa; así como también 
he visto a las personas que van con la ropa más elegante, profesionista o empresaria, pero yo creo que en su mayoría son mujeres y amas de casa, mujeres ya casadas" (Funcionario público en entrevista, 2014).

En México, no existen estadísticas que den cuenta de la participación de mujeres y hombres en prácticas de juego en casinos. Sin embargo, de acuerdo con diferentes estudios desarrollados en diversos países, son los hombres quienes asisten más a dichos espacios. Becoña (1997) sustenta que el perfil del jugador es ser hombre, entre 18 y 30 años y de baja escolaridad. Por su parte, Legarda, Babío y Abreu (1992), coinciden en señalar que son hombres, entre 31 y 57 años, casados con trabajo e ingreso fijo. Además, en un estudio desarrollado en Tegucigalpa, Honduras, $78 \%$ de los asistentes son del sexo masculino (Cruz et al., 2010). Por su parte Urquilla (2011) sustenta que el perfil del jugador de casino, en el Ecuador, es indistintamente hombre o mujer. Sin embargo, como señalan Levine Goldner, Rey Carrillo y Velázquez Nissán (2013) el juego ha cambiado a través de los siglos. Así, la adicción al juego, antes era considerada como una conducta masculina, mientras que en la actualidad un mayor número de mujeres en edad adulta se han incorporado a los casinos, pasando largas horas del día jugando en dichos espacios. De acuerdo con Cruz et al. (2010) los hombres tienden a jugar por excitación y ganar dinero y las mujeres para hacer frente a problemas personales como la soledad o la depresión, pero faltan estudios longitudinales que den cuenta de dicha diferencia. En Mérida, una ciudad básicamente tradicional, quizá la asistencia a los casinos esté relacionado con ser un espacio de interacción social entre las mujeres adultas jóvenes amas de casa, como un espacio nuevo que no está conquistado únicamente por los varones. Por otro lado, podría ser un espacio de entretenimiento importante para mujeres adultas jóvenes en virtud de que no existen muchos espacios de esparcimiento para ellas.

Otro sector poblacional que destaca como parte de su representación es el de personas adultas mayores. En este sentido, el pastor señala “...la gente que ha trabajado ahí, me ha dicho que va mucha gente anciana, me cuentan que han caído en esos lugares y que van diario, 
diario, diario y los días en los que más están, coinciden con los días de pago de la jubilación". Y por su parte, la diputada comenta: "los casinos están dando espacio a las personas de la tercera edad, pues aunque tengan humildes pensiones, la mayoría de los viejitos van y hasta ganan...más que la verdad, también es una guardería de adultos, pues los hijos los dejan ahí, y regresan por ellos varias horas después, pero estas personas no se sienten abandonadas, están encantadas de ir alli”. Cruz et al. (2010) en su estudio desarrollado en Honduras encontraron que el 48\% de los asistentes son personas entre 20 y 29 años y el 38\% es población mayor de 50 años. De ahí que la población adulta mayor se considere como un sector con alta presencia en los casinos. Es interesante cómo en la representación social de la diputada participante resalta el considerar el casino como "...una guardería de adultos, pues los hijos los dejan ahí, y regresan por ellos varias horas después; pero estas personas no se sienten abandonadas, están encantadas de ir alli’”. Representación social que ha sido promovida a través de medios de comunicación impresos con titulares como "casinos se convierten en estancias para ancianos solos" (Díaz, 24 de enero de 2014); amas de casa y 'abuelos' clientes asiduos a casinos (Vargas, 18 de enero de 2015); Ludopatía, la esclavitud de la tercera edad (Rojas, 18 de noviembre de 2012). Sin duda la tercera edad no tiene muchas opciones de esparcimiento ni de convivencia, por lo cual los casinos quizá cumplan esta función.

Desde la representación social de los participantes, el sector juvenil no se considera como un grupo poblacional involucrado en dichas prácticas. En esa lógica, el sacerdote señala que, desde su visión, "los jóvenes todavía no están metidos en los casinos. Desde este trabajo, yo no veo que haya mucho movimiento o que se note o haya noticias de jóvenes en casinos. De los 30 años para arriba sí, se nota y de 50 para arriba más" (En entrevista, 2014). Sin embargo, es importante señalar que a partir de enero de 2015 en México se estableció la edad de 21 años, como la mínima para ingresar a un casino, con la intención de prevenir la ludopatía en la juventud. Esta iniciativa es resultado de las Nuevas Reglas de la Ley de Juegos y Sorteos en México, aprobada en 2014. En muchos países occidentales, como México, donde la 
juventud y la fuerza física son altamente valoradas no debe de asombrarnos que la mayor parte de la oferta de actividades sociales, culturales y deportivas estén dirigidas a ellos y ellas. Sin embargo, los casinos en la actualidad intentan ganar terreno en este sector a través de ofertar actividades como conciertos de artistas jóvenes, concursos de belleza, de fisicoconstructivismo, entre otros.

Con respecto al estrato social, es interesante notar cómo la representación social dominante señala que la clase media trabajadora del norte de la ciudad es el sector de mayor asistencia y que miran al casino como un espacio para relacionarse, descansar y divertirse. En este sentido un funcionario municipal describe al casino como un "entorno social muy consolidado” y por su parte el sacerdote expresa: “...yo creo que la gente piensa, 'ya estuve trabajando mucho y me lo merezco, mis descansitos' y como se les hace muy divertido y no tienen que preocuparse de viajar a Cancún u otras partes, y aquí, a diferencia de otras partes, hay todavía una relativa seguridad" (En entrevista, 2014). La mayoría de los que van a los casinos son del norte simplemente rarísima gente del centro irá. De acuerdo con Urquilla (2011), en el Ecuador, los jugadores generalmente son profesionales, el $89 \%$ con ingresos medios y altos, el $51 \%$ con estudios universitarios o profesional y el 55\% son empresarios. Sin embargo de acuerdo con Tobón Álvarez, Cano Bedoya y Londoño Arredondo (2010), el juego patológico se distribuye de igual manera por todas las clases sociales. La población blanco de los casinos muy poco probable sea la clase alta, la cual seguramente prefiere asistir a casinos de otros países, como Las Vegas; o la clase baja, que seguramente podría asistir en muy pocas ocasiones y en poco tiempo. La clase media sin embargo sí podría darse esos espacios que algunos entrevistados señalan como un premio o un espacio merecido por su arduo trabajo. No es coindicdencia que en Mérida la mayoría de los casinos se encuentren en las plazas o centros comerciales donde asisten principalmente las personas, hombres y mujeres, de clase media.

Con respecto a las prácticas religiosas, es interesante señalar que tanto el sacerdote como el pastor dejan abierta la posibilidad de que personas cristianas evangélicas o católicas asistan a dichos espacios de 
juego. En dicho sentido, el sacerdote señaló “...yo creo que mucha gente muy religiosa incluso muy apostólica, muy metida con servicio a la iglesia que van, pero si hay gente religiosa que se inhiben, que no va no por cuestión del miedo, sino por la convicción" (En entrevista, 2014). Por su parte el pastor expresó que de su congregación no sabe de algún miembro que asista al casino; sin embargo, reconoce la posibilidad de que así sea, pues señala que sería difícil que se lo compartieran. En Honduras se encontró que el $57 \%$ de los asistentes a casinos son católicos y el 17\% evangélicos (Cruz et al., 2010). El aspecto religioso y el esparcimiento no son espacios contrarios o contradictorios. El uso diferenciado sobre la asistencia a los casinos relacionados con la religión podría relacionarse con dos aspectos importantes: (1) el aspecto de control social, donde algunas religiones o denominaciones religiosas pueden ser más estrictas con la asistencia a ciertos espacios y (2) la oferta de actividades de la congregación religiosa a la que se pertenece. Algunas denominaciones religiosas tienen diferentes clubs, grupos y actividades que pueden satisfacer las necesidades de interacción, esparcimiento y diversión de los hombres y mujeres que pertenecen a ella.

\section{Los clientes: características psicosociales y motivacionales}

En relación a las características atribuidas a los clientes, la característica más común con la que los participantes los representan es la soledad, independientemente de su postura moral, ética y política. Sin embargo es interesante destacar que lo que se entiende por soledad puede ser diferente entre ellos, lo cual sí se relaciona directamente con su posición. Por ejemplo, el pastor resalta la soledad desde un punto espiritual. Relaciona a los clientes de los casinos como gente con soledad interior. Destacando la necesidad de llenar vacíos a través de emociones como las que provocan el riesgo, la adrenalina de ganar, etc. En este sentido apunta:

...quienes asisten al casino son personas con enorme soledad interior, que solamente Dios puede llenar. Son personas que no están buscando cómo ganar, están buscando llenar el vacío interior. Además, yo considero que les emociona el riesgo, ya no es suficiente el comer 
mucho, el sexo, buscan algo más emocionante... los casinos te dan la sensación de bienestar, pero lo mismo te pueden hacer en la Iglesia, por lo que hay que razonar para decidir (En entrevista, 2014).

Adicionalmente, el pastor asocia el vació existencial con el consumo de alcohol y cigarro. Postura que encuentra coincidencia con el terapeuta participante, quien señala con base en su experiencia clínica que se ha encontrado con la presencia del juego en casinos acompañada de diferentes adicciones. En este sentido comenta: “... hay personas que pueden pasar de ser adictas o consumir algún tipo de sustancia al juego, y si dejan el juego pasan a otra, como una necesidad de tener algo en lo que tengan el control, que después termina no siendo así, como si se moviera nada más el objeto del hábito" (En entrevista, 2014).

El uso de sustancias como ya se mencionó es asociado frecuentemente con la asistencias a los casinos. Los casinos, como centros de entretenimiento, son lugares donde las bebidas alcohólicas y los alimentos son eje central de su publicidad o de los motivos de asistencia, sobre todo de jóvenes o adultos jóvenes.

En relación con el juego adictivo, para Becońa (1997) existen dos tipos de juego: el juego por ocio o placer y el juego adictivo. El primero es definido como una actividad normal en la vida de cualquier persona; sin embargo, hay un juego, el de azar, que puede traer graves problemas y consecuencias en un porcentaje significativo de personas y puede convertirse en una adicción. Convertirse en juego patológico.

De acuerdo con Sánchez Hervás (2003) el juego patológico comparte muchas características de las adicciones a sustancias. En un estudio realizado en Tegucigalpa se encontró que el 64\% de los jugadores consumen cigarro, el 70\% consumen bebidas alcohólicas, el 3\% marihuana y el $2 \%$ cocaína (Cruz et al., 2010). Sin embargo, aún se requiere de mayor detalle en la investigación acerca de la relación entre problemas de alcohol y problemas de juego (Ruíz-Pérez, 2009). De acuerdo con Gisbert y Calabuig (2004) ambas problemáticas pueden tener algún factor común o podría presentarse un aumento en el consumo de alcohol posterior a una acumulación de pérdidas en el juego y su impacto familiar o laboral o, el consumir alcohol antes de la conducta de juego 
podría proporcionar al sujeto cierta confianza sobre las posibilidades de ganar, por el efecto inicial desinhibidor del alcohol.

Por su parte el sacerdote representa a los clientes de casinos como personas "light", personas que en palabras de él "andan buscando oportunidades de diversión y a lo mejor de conseguirse un dinero fácil”; además de que las caracteriza como gente simpática, atractiva, sociable, aventurera y sin valores de solidaridad. Esta representación puede ser comprendida desde los planteamientos de la modernidad líquida de Bauman (1993) en la que los modelos y estructuras sociales ya no perduran lo suficiente como para enraizarse en los ciudadanos y vivimos bajo el imperio de la caducidad y la seducción.

El director de la asociación civil, de igual manera, caracteriza a los clientes como personas solas, que tienen mucho tiempo libre y que necesitan llenar esos espacios de convivencia y no saben cómo afrontar, por ejemplo la jubilación:

...no tienen las habilidades para afrontar ciertas situaciones como por ejemplo la jubilación, cuando los hijos se van creo que también coincide la edad de las personas que son adultas con la partida de los hijos y con el reencuentro de la pareja y que muchas veces es difícil; no se tienen los recursos psicológicos para poder manejar estas situaciones; de ahí que hay investigaciones de personas con pobre control de impulsos, por ejemplo (En entrevista, 2014).

Esta representación encuentra coincidencia con estudios realizados por Wagner, Myers y Ininch (1999) y Finney y Moos (1995) quienes comprobaron que, a diferencia de los adultos mayores, son los adolescentes y adultos quienes utilizan estrategias de afrontamiento adecuadas y presentan menores adicciones y consumos que quienes utilizan estrategias de evitación.

Otra característica de los clientes y asistentes a los casinos se relaciona con percibirlas como obsesivas y compulsivas. De acuerdo con el Manual D.S.M, IV-TR de la American Psychiatric Association APA (2001) el juego patológico se le consideraba como un trastorno por falta de control de impulsos. Sin embargo, a diferencia del anterior, 
en la última revisión del Manual Diagnóstico y Estadístico de los Trastornos Mentales D.S.M. V American Psychiatric Association APA (2013) se considera como un trastorno adictivo. Al respecto, Arbinaga (2000) encontró niveles de depresión leve o moderada en el 54.6\% de las mujeres con algún problema de juego y en el $37.5 \%$ en las jugadoras patológicas. No obstante, acerca de la relación entre trastornos mentales y juego patológico, Estévez y Calvete (2007) sostienen que falta claridad sobre qué tipo de trastornos se asocian con el juego patológico y sobre cuál es la naturaleza de esa relación.

Sin embargo, a pesar de que existe poca investigación en este sentido, sobre todo en algunos países como México, sorprende cómo la representación social de la práctica de juego en casinos está cargada de elementos patologizantes, morales y éticos. En este sentido, el director de una asociación civil comenta:

...la misma sociedad empieza a estigmatizar al jugador, no como un enfermo, si no como un vicioso y entonces hay una carga moral, entonces ese es una persona negativa, es una persona mala, es una persona irresponsable, porque les está robando, porque es un desobligado, porque es un irresponsable (...) lo ve como una irresponsabilidad, como un vicio, como algo malo, entonces lleva una carga moral, igual con el adicto a sustancias, antes de verlo como un enfermo lo ven como un delincuente, como un vicioso, pero habrían que preguntarnos cómo la misma sociedad ha contribuido con una tolerancia y permisividad ...si te la vas a pasar bien adelante, pero ya cuando aparecen los problemas, ya cambia mi actitud y cambia mi percepción (En entrevista, 2014).

En los párrafos anteriores podemos notar con claridad cómo las posturas ante dicha práctica varían según el rol del representante entrevistado. De tal manera que los representantes religiosos asocian la práctica de juego en casinos con el vacío existencial y quienes están enfocados en el campo de la salud lo asocian con características de personalidad y patología. Aunque es claro que todos los participantes representan a las personas jugadoras como personas con problemas de juego y con vivencias de soledad. 


\section{El casino: espacio de recreación y adrenalina}

De acuerdo con Korn y Shaffer (1999) el consumo de juegos de azar puede tener consecuencias negativas como la adicción, pero también positivas como la generación de integración social, el entretenimiento o recreación y la reducción de ansiedad y estrés. El casino se considera un espacio abierto al que puedes acudir a cualquier hora. Desde la mirada de los y las representantes participantes, el casino es un espacio social de recreación. En este sentido comenta un psicólogo:

...de entrada es un lugar de recreación, es un lugar para pasar el tiempo, es un lugar para algunos para hacer amigos, convivir, socializar pero, algo pasa en la vida de estas personas, en la conducta de esta personas que esta práctica o esta conducta que inicialmente puede parecer, o es de hecho, saludable pueda convertirse en un problema, aquí si tenemos que valorar una serie de situaciones, factores, pero de entrada yo pensaría que son lugares para que la gente vaya a distraerse, como puede ser un estadio, como pueden ser los bolos, como puede ser el cine, como puede ser una biblioteca (En entrevista, 2014).

Esta representación coincide con un estudio realizado en Chile por Visión Humana (2011) en que al entrevistar a 1385 personas jugadoras en casinos, el $81 \%$ señaló como la principal razón para asistir al casino es el poder entretenerse. Adicionalmente, en dicho estudio, destacan el $38 \%$ con el salir de la rutina haciendo algo distinto, el $34 \%$ con el buscar ganar dinero y el $30 \%$ con el desconectarme del día a día. Por su parte en un estudio desarrollado en Ecuador por Uquillas (2011) el 59\% lo mira como oferta de diversión, el $32 \%$ de recreación y un $12 \%$ cree que es oferta turística; adicionalmente, el $70 \%$ de los jugadores consideran que el juego en casino es de entretenimiento, el 20\% de esparcimiento y apenas el $3 \%$ cree que los casinos son lugares de asocia de gente mala. En este sentido, los casinos en Mérida podrían ser considerados como centros de diversión y esparcimiento. No todos los asistentes resultan jugadores patológicos, ni todos los asistentes juegan. Muchas veces las personas asisten a los casinos por sus restaurantes $24 / 7$, por los espectáculos que ofrecen o por sus precios módicos en bebidas y alimentos. 
Otra de las representaciones asociada al casino es la adrenalina que dicho espacio genera. En este sentido, se exponen las siguientes citas: "Yo podría decir que la adrenalina que sienten es lo que les hace ir a esos lugares, tienen la esperanza de ganar" (pastor, en entrevista, 2014), “...como los jugadores dicen: cuando estoy en el casino me siento vivo porque siento una adrenalina en mí y que eso me hace sentir vivo, entonces se activan ahí los sistemas de recompensa del cerebro, no me estoy metiendo sustancias, pero si me estoy metiendo esta relación con el juego" (director de asociación civil, en entrevista, 2014), “... a algunas personas la deuda no les preocupa, es más la adrenalina que sienten al jugar. En el caso de los hombres, ellos dicen que, les da mucho placer estar ahí, la adrenalina, ellos hablan de adrenalina, de que la adrenalina de estar ahí, de jugar de todo, les produce esta sensación de sentirse muy bien [...] y que les produce mucho placer..." (psicólogo, en entrevista, 2014). En el estudio desarrollado por Visión Humana (2011), el 22\% de los jugadores señalaron como razón para asistir al casino, el sentir emoción y adrenalina. Además de que el 38\% de los jugadores potenciales manifiestan su acuerdo con dicha motivación.

La asistencia al casino y el juego de azar, desde la mirada de los representantes entrevistados, no se asocia a la expectativa de ganar dinero. De acuerdo con la mayoría de los representantes entrevistados, no se juega por dinero, muchas veces se juega por placer. En este sentido un psicólogo comenta:

Desde mi experiencia nadie lo ve como una cuestión de dinero, que curiosamente es voy al casino porque voy a ganar, en los clientes que yo he atendido no es una cuestión de dinero, porque al final de cuentas siempre saben que están perdiendo dinero, porque si sacan sus cuentas, si he perdido pero me da esto y lo otro... En ninguno de los casos con hombres o con mujeres he escuchado que tiene que ver con dinero, bueno cuando ganan se sienten muy felices y todo mundo les hace fiesta y eso es algo padre, pero tiene que ver con el acercamiento, tiene que ver con toda la fiesta que la gente hace (En entrevista, 2014). 
De acuerdo con Garrido Palacios (2005) el reto que supone el juego lo llena de adrenalina y la necesidad de jugar supera toda la lógica. No se trata de ganar, sino realmente de jugar, por lo tanto, ganar no es la finalidad. Cuanto más se juega más necesidad se tiene de seguir jugando, se gane o se pierda. Sin embargo, en el estudio de Visión Humana (2011) el buscar ganar dinero se consideró como una razón de asistencia al casino por el $34 \%$ de los jugadores y por el $43 \%$ de los jugadores potenciales. Tomar riesgos también significa estar vivo, quizá es una respuesta a que algunos de los/las asistentes a los casinos quieran salirse de su rutina laboral o familiar. De percibirse $-o$ creer que pueden ser percibidos por otros asistentes- como sujetos a quienes no les importa perder porque pueden recuperar sus pérdidas y ser autosuficientes.

\section{El casino: espacio de convivencia, pertenencia y estatus}

De acuerdo con la representación social de los participantes, el casino es un espacio de convivencia social que posibilita a sus clientes el cambio de sus rutinas cotidianas. En este sentido comenta el funcionario de gobierno:

... cuando no había casinos en Mérida, tú tenías un ritmo cotidiano de vida, a lo mejor ir a recoger a tus hijos, llevarlos al béisbol, etc., se abre esta opción y tú por curiosidad, ocio o invitación asistes al casino y te encuentras en él un lugar donde te gusta estar, la pasas bien, conoces gente. Esto te abre la posibilidad porque no podemos satanizar a los casinos, porque los casinos son nada más un centro de diversión, porque el que tiene el problema no es el casino, yo creo que es la persona (En entrevista, 2014).

Adicionalmente, desde la representación social de los representantes religiosos, el casino es un espacio que "brinda" estatus social a sus clientes. En palabras del pastor participante: "en los casinos se desarrolla una cultura como que 'eres algo especial', como que eres de 'clase', jajaja, unos lo ven como un lugar de elite, que realmente no lo es. Yo creo que hay gente que lo ve como de prestigio...ver que puedo lograr y alcanzar". Por su parte el sacerdote apunta que los casinos funcionan como 
espacios para conocer gente con poder adquisitivo alto y establecer relaciones amorosas. En este sentido señala: “... claro que el estatus influye; si una mujer allá puede encontrar a un tipo que tiene mucha lana, y si la mujer está todavía solterona grandecita y el varón la encuentra interesante y él a ella, pues sirve para eso, para encuentros amoroso y para ver quién es buen partido, por el estatus social”. En los casinos lo que está en juego es el dinero. El prestigio y el estatus normalmente están directamente relacionado con el dinero que posees, las cosas que posees, los símbolos de estatus que se obtienen, los lugares a los que se asiste, etc. Todo lo anterior es aprovechado por los casinos, quienes a través de sus máquinas, mobiliario, colores, uniformes, etc, intenta mostrar que al asistir al casino entras a un mundo de lujo y comodidad, donde como huésped eres atendido con respeto y amabilidad.

Por su parte, el casino se representa como un espacio que aporta a la construcción de procesos identitarios, particularmente para las mujeres. De acuerdo con un terapeuta participante la incursión de las mujeres al juego en casinos les ha posibilitado crear nuevas relaciones sociales y construir un mundo personal alrededor del juego, dejando de lado, por alunas horas, su rol de madre de familia. En este sentido, un psicólogo, comenta:

... una de las cosas que he estado escuchando que ocurre en los casinos es esta parte sobre todo en el caso de las mujeres dicen que el ir al casino les permite tener un mundo personal, un mundo donde todo el mundo se conoce, en donde llegan y te saludan donde eres alguien, donde por ejemplo ganas en las maquinitas y todo el mundo te felicita, te hace fiesta y ellas se sienten como muy contentas (En entrevista, 2014).

... tienen amigas con quienes comparten algo muy en específico, les genera como una pertenencia a un grupo, tienen todo un lenguaje común que les hace sentirse muy cómodas... en algunos casos las mujeres dicen, si de repente llego a la casa mis hijos ya se fueron, o sea mis hijos andan de no sé qué, mi marido no llega porque tiene trabajo porque está fuera, qué hago, qué hago yo, entonces me voy al casino y me encuentro con gente que conozco (En entrevista, 2014). 
Hoy, los casinos ofrecen a sus consumidores el trascender la rutina de la cotidianeidad; el placer inmediato, la esperanza de recompensa, estados paratélicos, la posibilidad de tomar riesgos poco amenazantes y fugaces, la evasión y el desahogo, ocupan un lugar prioritario (Echeverría Echeverría \& Evia Alamilla, 2013).

... este espacio que les permite tener relaciones que no tienen de otra manera, más que las relaciones que tienen a nivel de ser esposa, de ser hija, de ser mamá por ejemplo, el hecho de que se van al casino les da este espacio de recreación y de relaciones que no tienen en ningún otro lugar. Hay quienes dicen, me voy a comer allá, comen, y bueno pues te encuentra a todo mundo y juega y esto y va siendo una sensación una emoción un sentimiento que les va produciendo placer y les produce placer a un nivel de las relaciones que están estableciendo y de pertenencia. Ahí tengo a mis amigas, ahí tengo gente, puedo ir a cualquier hora, acá siempre hay gente que te conoce siempre hay gente que te trata bien, siempre hay gente que se emociona con que ganes (En entrevista, 2014).

El casino es un lugar accesible, está abierto a todos quienes sean mayores de edad, cuenten con una identificación, un mínimo de 50 pesos y el tiempo para esta actividad. No importa si eres joven o viejo, flaco o gordo, rico o pobre, blanco o moreno, solo o acompañado puedes participar en dicho espacio. Los casinos ofrecen a sus clientes la sensación 'de ser respetados' en su diversidad, de ser espacios que 'favorecen la inclusión social', tan necesitada en las sociedades contemporáneas (Echeverría Echeverría \& Evia Alamilla, 2013). En este sentido, comenta la diputada participante:

...los casinos están dando espacio a las personas de la tercera edad, Además, lo mismo ves a una maestra de ascendencia árabe que a una humilde maestra. Se vive la democracia plena. Está abierto para todos. He visto, sobre todo, gente mayor, humilde, lo ves por la forma de vestir y de llegar (en taxi). Eso sí que ni que, los casinos es un sitio democrático. Hay respeto para no fumadores, pero también los fumadores tienen su espacio. Hay suficiente espacio para que se pueda mover la gente discapacitada. Es un espació que cumple las 
características de ser un espacio incluyente, democrático y accesible (no necesitas mucho dinero para estar allí). Nunca te van a negar la entrada como sí sucede en otros lugares por ejemplo los antros a dónde van los muchachos. Aquí por el contrario, todo mundo es bienvenido. Nunca he oído de maltratos o conductas discriminatorias. Lo mismo pueden ir homosexuales, la mujer humilde que la encuentras sentada junto a la mujer que lleva las joyas de la corona y no pasa nada desagradable (En entrevista, 2014).

\section{Conclusiones}

De acuerdo con Perera (1999) las funciones de las representaciones sociales son de conocimientos, identitaria, orientadora y justificatoria. En este trabajo se evidencia cómo las representaciones, en su función de conocimiento, permiten a los actores sociales integrar sus conocimientos, de modo comprensible para ellos, coherente con su forma de pensar, su formación profesional, los valores, la moralidad y la ética.

Es interesante notar cómo las representaciones sociales participan en la definición de la identidad de cada uno de los representantes participantes, salvaguardando la identidad del grupo al que representan. Situación que les permite la elaboración de una identidad social compatible con el sistema de normas y valores social e históricamente determinados.

Adicionalmente, es importante resaltar cómo las representaciones sociales de los diferentes representantes guían sus comportamientos y prácticas, permitiendo producir un sistema de anticipaciones y expectativas, que constituyen una acción sobre la realidad. Lo anterior define lo que es considerado como lícito y tolerable en un contexto o institución social dada. Finalmente, las representaciones permiten justificar una posición.

Las experiencias de los entrevistados nos invitan a la reflexión acerca de cómo socialmente se ha generado un discurso cada vez más presente y poderoso sobre el juego en casinos que acentúa el papel del riesgo y la patología, y que se ha convertido en un modo de evaluar y condenar no oficialmente este tipo de conducta. Donde las instituciones han jugado 
un papel doble por un lado promoviendo y facilitando la apertura de casinos y por otro creando a través de los profesionales un discurso que sanciona la misma conducta. Y que la popularización de dicho discurso desvincula a las instituciones de este doble papel y su responsabilidad en ello ya que el encargo condenatorio queda en la sociedad.

Con la intención de promover una comprensión más integral de los juegos de azar en casinos, hace falta superar las miradas que limitan el conceptualizarlos en franca asociación con la patología. El fenómeno de los juegos de azar en casinos implica muchos otros aspectos que no debemos pasar por alto si queremos promover una comprensión más amplia, compleja y completa de este fenómeno social y, por supuesto, de cualquier otro (Echeverría Echeverría \& Evia Alamilla, 2013). El establecimiento de los casinos es una realidad social, clausurar la vocación de entretenimiento que como espacio social poseen resulta por demás limitado y limitante. Lo verdaderamente importante $-y$ urgente- sería reflexionar y legislar sobre ellos, con la intención de potenciar sus elementos positivos y minimizar sus efectos negativos.

Los juegos de azar se han visto y se continúan mirando negativamente desde una perspectiva moral, debido al gasto de dinero que dicha práctica conlleva y de las asociaciones con las drogas, el alcohol, la prostitución, etc. Sin embargo, es interesante notar cómo el uso extensivo y en aumento de las tarjetas de crédito, las hipotecas, los préstamos para la adquisición de bienes y servicios ajenos a la satisfacción de necesidades básicas, no se interpretan de la misma manera. Aunado a que son estas estrategias comerciales las que aportan dinamismo a los juegos de azar, sin tener dinero, puedo acceder a él, por ejemplo, desde una tarjeta de crédito y así seguir jugando. Socialmente es más desvalorizado jugar en exceso que ser deudor crediticio; al grado que la ludopatía está clasificada como problema a nivel internacional a diferencia de las deudas crediticias, que hasta el momento actual no se miran de la misma forma (Echeverría Echeverría \& Evia Alamilla, 2013).

De acuerdo con Cruz et al. (2010), la ausencia de estudios particularmente en la región latinoamericana, constituye un reto. De ahí la pertinencia de continuar generando procesos investigativos que 
permitan ampliar la concepción del fenómeno de los juegos en casinos, discutir los conceptos de juego responsable y juego saludable, generar datos que den cuenta de la situación actual, dar seguimiento y evaluar las estrategias de prevención de la ludopatía que se están desarrollando en nuestro país, desarrollar trabajo en la línea de la promoción de la salud, regularla las prácticas mercadológicas y asegurar que sus mensajes sean realistas y no ofrezcan imposibles a las personas e impactar en las políticas de regulación y en los servicios que se ofrecen a la ciudadanía en materia de salud, desarrollo social y economía.

\section{Referencias}

American Psychiatric Association (APA). (2001). Manual diagnóstico y estadistico de los trastornos mentales D.S.M, IV-TR. Barcelona: Masson.

American Psychiatric Association (APA). (2013). Manual diagnóstico y estadístico de los trastornos mentales D.S.M, V-TR. Washington: APA. Arbinaga, F. (2000). Características sociodemográficas, consumo de drogas, depresión y juego patológico en un grupo de mujeres de Punta Umbra (Huelva): un estudio descriptivo. Anales de Psicología, 16(2), 123-132.

Bauman, Z. (2003). Modernidad líquida. México DF: Fondo de Cultura Económica.

Becoña, E. (1997). Características de la mujer patológica. Revista de Psicopatología y Psicología Clínica, 2(1), 21-34. España. Disponible en: http://e-spacio.uned.es/fez/eserv.php?pid=bibliuned: Psicopat-1997-628F1696-6418-0DE9-5669-192EA9247847 \&dsID=PDF

Carrillo Trujillo, C.D. (2013). Casinos. Aspectos económicos y políticos. En R. Echeverría Echeverría (Coord.), Juegos de azar en casinos reflexiones en el contexto contemporáneo (pp. 79-100). Mérida Yucatán: Universidad Autónoma de Yucatán.

Cruz, A., Argeñal, A., Padilla, C., George, E., Blanco, E., Novondo, F., Padilla, G., Galo, G., Ortega, N., Galeano, R., Paz, Y., 
Zavala, G. \& Nuñez, R. C. (2010). Ludopatía: características de la población que asiste a salar de juego en Tegucigalpa. Revista Facultad de Ciencias Médicas, 27-37. Disponible en: http://www. bvs.hn/RFCM/pdf/2010/pdf/RFCMVol7-2-2010-5.pdf

Díaz, C. (24 de enero de 2014). Casinos se convierten en estancias para ancianos solos Milenio Novedades. Disponible en: http://sipse. $\mathrm{com} / \mathrm{milenio/casinos-en-merida-se-convierten-en-estancias-}$ para-ancianos-solos-72329.html

Echeverría Echeverría, R. \& Evia Alamilla, N.M. (2013). Algunas consideraciones finales en torno a los juegos de azar en casinos. En R. Echeverría Echeverría (Coord.), Juegos de azar en casinos: reflexiones en el contexto contemporáneo (pp. 183-194). Mérida Yucatán: Universidad Autónoma de Yucatán.

Echeverría Echeverría, R. \& Roa Muñoz, V. R. (2013). Los juegos de azar en casinos ¿Problema social o individual? En R. Echeverría Echeverría (Coord.), Juegos de azar en casinos: reflexiones en el contexto contemporáneo (pp. 121-154). Mérida Yucatán: Universidad Autónoma de Yucatán.

Elejabarrieta, F. (1995). Las representaciones sociales. En A. Echevarría, Psicología social sociocognitiva. Bilbao: Desclée de Brouwer, S.A.

Estévez, A. \& Calvete, E. (2007). Esquemas cognitivos en personas con conducta de juego patológico y su relación con experiencias de crianza. Clínica y Salud, 18(1), 23-43.

Finney, J.W. \& Moos, R.H. (1995). Entering treatment for alcohol abuse: A stress and coping model. Addiction, 90, 1223-1240. https://doi.org/10.1111/j.1360-0443.1995.tb01092.x

Foucault, M. (1996). Historia de la sexualidad, la voluntad del saber. México: Siglo XXI.

Fuentes Gómez, J.H. (2013). Contexto sociocultural de los casinos en el espacio urbano actual. En R. Echeverría Echeverría (Coord.), Juegos de azar en casinos: reflexiones en el contexto contemporáneo (pp. 17-46). Mérida Yucatán: Universidad Autónoma de Yucatán. Garrido Palacios, P. (2005). Ludopatía o adicción al juego. El divan, 227-232. Disponible en: http://www.google.com.mx/url?sa=t\& 
$\mathrm{rct}=\mathrm{j} \& \mathrm{q}=\&$ esrc $=\mathrm{s} \&$ frm $=1 \&$ source $=$ web $\& \mathrm{~cd}=26 \& \mathrm{ved}=0 \mathrm{CDkQ}$ FjAFOBQ\&url=http\%3A\%2F\%2Fdialnet.unirioja.es\%2Fdes carga\%2Farticulo\%2F3099532.pdf\&ei=xpaVd7hA4vCsAXa6I CoBg\&usg=AFQjCNEvnGAFQ11Q262uYsf2GPcHHVq_Hw Gisbert y Calabuig, M. (2004). Medicina legal y toxicología. Barcelona: Masson.

Jodelet, D. (1991). La representación social: fenómeno, concepto y teoría. En S. Moscovici, Psicología Social II. Barcelona: Paidós.

Korn, D.A. \& Shaffer, H.J. (1999). Gambling and the health of the public: Adopting a public health perspective, Journal of Gambling Studies, 15(4), 289-365. https://doi. org/10.1023/A:1023005115932

Legarda, J. J., Babío, R. \& Abreu, J.M. (1992). Prevalence estimates of pathological gambling in Seville (Spain). British Journal of Addictions, 87(5), 767-770. https://doi. org/10.1111/j.1360-0443.1992.tb02722.x

Levine Goldner, J., Rey Carrillo, L. S. \& Velázquez Nissán, A. (2013). Ludopatía en mujeres: un estudio de caso. Alternativas en Psicologia, XVII(28), 33-40.

McGurrin, M. C. (1992). Pathological gambling: Conceptual, diagnostic, and treatment issues. Sarasota, Florida: Professional Resource Press. Moscovici, S. (1981). La era de las multitudes. Un tratado histórico de psicología de masas. México: Fondo de Cultura Económica.

Perera, M. (1999). A propósito de las representaciones sociales: apuntes teóricos, trayectoria y actualidad. Informe de investigación. CIPS. La Habana.

Perera, M. (2005). Sistematización critica de la teoría de las Representaciones Sociales. (Tesis doctoral). Centro de Investigaciones Psicológicas y Sociológicas. Ciudad Habana, Cuba.

Rodríguez Gómez, G., Gil Flores, J. \& García Jiménez, E. (1999). Metodología de la investigación cualitativa. Granada: Aljibe.

Rojas, A. (18 de noviembre de 2012). Ludopatía, la esclavitud de la tercera edad. Clinico.cl Disponible en: http://clinico.cl/2012/11/18/ ludopatia-en-la-tercera-edad/\#sthash.3kU26d2k.dpbs. 
Ruíz-Pérez, J.I. (2009). Juego patológico en usuarios de casinos en Bogotá: prevalencia y relaciones con consumo de alcohol, búsqueda de sensaciones y patrones de juego. Disponible en: http:// www.bdigital.unal.edu.co/15387/2/9993-141754-4-PB.html

Sánchez Hervás, E. (2003). Juego patológico: un trastorno emergente. Trastornos Adictivos, 5(2), 75-87. https://doi.org/10.1016/ S1575-0973(03)78557-2

Tobón Álvarez, N. I., Cano Bedoya, V.H., Londoño Arredondo, N.H. (2010). Perfil cognitivo en personas con ludopatía: aproximación a la población no institucionalizada. Revista Virtual Universidad Católica del Norte. Disponible en: http://www.redalyc.org/articulo.oa?id=194214466007

Uquillas A. (2011). Diagnóstico y análisis del impacto de los casinos en el Ecuador. Disponible en http:/www.eumed.net/cursecon/ ecolat/ec/2011/au.html

Vargas, M. (18 de enero de 2015). Amas de casa y 'abuelos' clientes asiduos a casinos. Norte Digital. Disponible en: http://nortedigital. $\mathrm{mx} /$ amas-de-casa-y-abuelos-clientes-asiduos-a-casinos/

Visión Humana (2011). Radiografía de los jugadores de casinos de juegos. Disponible en: http://es.slideshare.net/visionhumana/ presentacin-estudio-radiografa-de-los-jugadores-de-casinos-dejuegos-2011

Wagner, W. \& Flores-Palacios, F. (2010). Apuntes sobre la epistemología de las representaciones sociales. Educación Matemática, 22(2), 139-162. Disponible en: http://www.scielo.org.mx/pdf/ ed/v22n2/v22n2a7.pdf

Wagner, E. F., Myers, M.G. \& Ininch, J.L. (1999). Stress-coping and temptation-coping as predictors of adolescent substance use. Addictive Behaviors, 24(6), 769-779. https://doi.org/10.1016/ S0306-4603(99)00058-1

Recibido: 16 de junio, 2015

Revisado: 06 de mayo, 2016 Aceptado: 10 de junio, 2016 